\title{
Respuesta electroquímica del cobre durante el fenómeno de corrosión por picaduras en soluciones con iones cloruro $^{\left({ }^{(0}\right.}$
}

\author{
E. García O. ${ }^{(*)}$, J. Uruchurtu ${ }^{(*)}$ y J. Genescá ${ }^{(* *)}$
}

\begin{abstract}
Resumen El cobre es un material frecuentemente empleado para la construcción de tuberías, sujeto a la acción de los cloruros, que ocasionan corrosión por picaduras. El presente trabajo busca determinar un posible mecanismo de corrosión por picaduras en el cobre por la acción de los cloruros, apoyándose en la información que ofrecen las técnicas electroquímicas de polarización, espectroscopía de impedancia faradaica y ruido electroquímico.
\end{abstract}

Palabras clave: Cobre. Picaduras. Corrosión localizada.

\section{Electrochemical response of copper during pitting corrosion phenomena in chloride solutions}

\begin{abstract}
Copper is a widely used material in water pipe systems. It is subjected to the action of chloride ions, which cause pitting corrosion. This work proposes a possible mechanism for the pitting corrosion of copper in chloride solutions. It was determined using electrochemical techniques such as polarization curves, impedance spectroscopy and electrochemical noise.
\end{abstract}

Keywords: Copper. Pitting. Localized corrosion.

\section{INTRODUCCIÓN}

El fenómeno de la corrosión por picaduras es una de las formas más dañinas de corrosión, tanto por su carácter localizado como porque no guarda una relación directa con la cantidad de material perdido. Por ello, es de gran importancia la realización de estudios sobre este problema.

El cobre es uno de los materiales más empleados en la construcción de tuberías, que comúnmente transportan soluciones acuosas con concentraciones variables de cloruros.

Es bien conocido que los cloruros son aniones asociados con el fenómeno de la corrosión por pica-

(•) Trabajo recibido el día 25 de abril de 1995.

(*) Dpto. de Fisicoquímica Aplicada. Instituto de Investigaciones Eléctricas (IIE). Avda. Reforma, núm. 133. Colonia Palmira. 62490 Temixco, Morelos (México).

(**) Facultad de Química. Universidad Nacional Autónoma de México (UNAM). Ciudad Universitaria. 04510 México D.F. (México). duras en diversos materiales, siendo el cobre uno de los afectados. Por esta razón, es importante determinar el efecto de este anión, evaluándolo por medio de técnicas electroquímicas, que informan de las condiciones en que se encuentra el material y de la evolución del mismo antes de que se presenten las picaduras.

Es común que el fenómeno de la corrosión por picaduras esté asociado con roturas de la capa pasiva. May (1) ha indicado que este mecanismo se puede presentar en el cobre durante este fenómeno de corrosión.

Esta circunstancia es muy importante, ya que la técnica de ruido electroquímico es muy sensible a esta situación. Gabrielli et al. (2) determinaron este efecto por medio de series de tiempo en corriente, donde se asociaban grandes fluctuaciones de corriente con la formación de picaduras.

Uruchurtu et al. (3) hicieron una importante aportación al estudio de las fluctuaciones del potencial o de la corriente durante el fenómeno de picadura, proponiendo que las componentes en alta 
frecuencia pueden ser los indicadores de la presencia de corrosión por picaduras, cuando ésta tiene su origen en un fenómeno de rotura y recuperación de la capa pasiva.

Se han propuesto diversos mecanismos para explicar el fenómeno de la corrosión por picaduras, entre los que resaltan los siguientes.

\subsection{Modelo de desplazamiento de ion disuelto}

Este modelo fue propuesto por Kolotyrkin (4) y Uhlig (5), y considera la formación de una capa pasiva por la adsorción de una monocapa de oxígeno en la superficie metálica. La picadura se inicia por la adsorción de aniones agresivos en sitios discretos sobre el metal, provocando el desplazamiento del oxígeno de la monocapa. Esto es el resultado de una adsorción competitiva entre el oxígeno y los aniones agresivos tales como los cloruros. El mecanismo considera la adsorción y desplazamiento en sitios discretos debido a la presencia de defectos cristalinos, inclusiones, etc. (6).

\subsection{Modelo de migración iónica o de penetración}

Este modelo propone el desplazamiento de aniones agresivos a través de la capa pasiva, por la migración iónica debida a un campo eléctrico, proceso similar al que acompaña a los cambios de conductividad de la capa pasiva (6-9).

Hoar et al. (9) sugieren la iniciación de la picadura como una adsorción de los aniones agresivos en la superficie de óxido, seguida de una penetración de los mismos a través de la capa pasiva. Esta adsorción se lleva a cabo en sitios donde el potencial se encuentra por encima del potencial crítico de picadura.

\subsection{Modelo de discontinuidad de capa}

Este modelo propone el origen en un ataque localizado por la presencia de poros o defectos existentes en la capa pasiva. Richardson et al. (10) sugieren que la iniciación de la picadura tiene lugar en defectos de la capa de óxido, y que estas fallas están siempre presentes cuando el metal está sumergido en un medio agresivo.

Estos mismos autores (10) distinguen entre defectos mecánicos y defectos residuales. Son defectos mecánicos los atribuidos a vacancias o dislocaciones, que emergen a la superficie del metal, mientras que los defectos residuales se deben a impurezas o a segregaciones. El potencial crítico de picado en este caso corresponde al potencial de adsorción de los aniones agresivos sobre el metal en ausencia de poros o de defectos en la capa pasiva.

La rotura inducida de la capa pasivante ha sido propuesta por pocos investigadores, entre los cuales Hoar (7) propone que existe una adsorción localizada de aniones agresivos en la superficie de la capa pasiva, reflejándose en un abatimiento de la tensión superficial, hasta que esto ocasiona la rotura de la capa pasiva en sitios discretos, provocándose así el inicio de la picadura.

Sato (11) sugiere la ruptura de la capa pasiva en sitios donde ésta tiene menor espesor, debido a la presión electrostática a la que está sujeta dicha capa pasiva. La presión electrostática se puede deducir a través de la siguiente ecuación:

$$
P-P o=\left(\left(e(e-1) E^{2}\right) / 8 \Pi\right)-(\gamma / L)
$$

donde:

$P=$ Presión electrostática sobre la capa pasiva

$P o=$ Presión atmosférica

$e=$ Constante dieléctrica de la capa pasiva

$E=$ Campo eléctrico

$\gamma=$ Tensión superficial

$L=$ Espesor de la capa pasiva

Estudios realizados por Sato (11) han estimado valores de $e$ entre 2 y $15, E$ entre $10^{6}$ y $10^{7} \mathrm{~V} / \mathrm{cm}$ y de $\gamma$ entre 10 y 500 dinas $/ \mathrm{cm}$. Cálculos realizados en el intervalo de valores antes indicado han arrojado valores de presión electrostática entre 10 y 100 - $\mathrm{kg} / \mathrm{cm}^{2}$, hecho que causa una deformación plástica sobre la película o la rotura de la misma. Aquí, el potencial crítico de picadura puede definirse como el potencial al cual la presión electrostática es lo suficientemente grande como para fracturar la capa pasiva.

\subsection{Modelo de formación de una capa salina}

De acuerdo con varios autores (12-14), la iniciación de la picadura se halla estrechamente relacionada con la formación de una capa salina entre el metal y la solución.

La coexistencia estable de un estado activo dentro de la picadura y de un estado pasivo en los alrededores de la picadura a un mismo potencial, sugiere la existencia de una barrera con una alta resistencia óhmica. Esta barrera puede consistir en el depósito de productos de corrosión en la boca de la picadura, o en la existencia de una capa salina presente en la superficie interna de dicha picadura.

Vetter et al. (12) consideraron la formación de una delgada capa salina de algún halogenuro que nuclea en la superficie de una capa de óxido no poroso, originándose en dicho sitio la iniciación de la picadura, donde se encuentra la evidencia de la 
existencia de dicha capa salina. A potenciales más positivos que el potencial crítico de picado, se incrementa la formación de dicha capa salina sobre la formación del óxido de la capa pasiva; en cambio, a potenciales inferiores sucede exactamente lo contrario.

Szklarska-Smialowska (14) propone que la nucleación de la picadura ocurre en sitios débiles de la capa pasiva, asumiendo la formación de una película salina.

Estos son algunos de los principales mecanismos propuestos para la iniciación de la picadura. Ello no quiere decir que sean los únicos o que alguno descarte al otro, pudiendo ocurrir que existan mecanismos de picado que combinen a los diferentes propuestos.

En este trabajo se presenta la respuesta electroquímica del cobre frente al fenómeno de la corrosión por picaduras en solución de $\mathrm{NaCl}$, utilizándose para su estudio diferentes técnicas electroquímicas con objeto de contribuir a un mejor conocimiento del mismo y poder proponer el posible mecanismo que se presenta en el fenómeno.

En diversos estudios (15) se ha encontrado que es necesaria una concentración mínima de cloruros para que se presente el fenómeno de la corrosión por picaduras en el cobre. Por esta razón, para el presente trabajo se ha seleccionado una concentración de 3 $\% \mathrm{NaCl}$, que es una concentración adecuada para que el cobre sufra corrosión por picaduras.

\section{DESARROLLO EXPERIMENTAL}

Para poder realizar los ensayos electroquímicos, se construyeron electrodos consistentes en el encapsulado en resina poliéster de una muestra de cobre comercial, del 99,94\% de pureza, exponiendo a la acción del medio una superficie de $1 \mathrm{~cm}^{2}$ del metal. Dicha superficie expuesta se lijó hasta un grano de 1.200 y se desengrasó con acetona, contando dicho electrodo con una conexión eléctrica aislada para su conexión a una interfase electroquímica Schlumberger 1286. Este equipo controla el potencial de la probeta con respecto a un electrodo de referencia. En este caso, se ha utilizado, como referencia, un electrodo de calomelanos saturado (ECS), y como contraelectrodo, una barra de grafito.

Como se indicó anteriormente, se utilizó un equipo de electroquímica Schlumberger 1286, con el que se aplicaron las técnicas de polarización y de ruido electroquímico.

Para la técnica de impedancia faradaica, a dicho equipo se le acopló un analizador de ganancia marca Schlumberger 1253.

Estos equipos electroquímicos se conectaron a un ordenador que los controlaba a través de la interface de comunicación RS232.
Los parámetros de las diferentes técnicas electroquímicas fueron: para curvas de polarización, se fijó una variación de potencial de $20 \mathrm{mV}$ cada $6 \mathrm{~s}$; para la técnica de ruido electroquímico, un muestreo cada $0,7 \mathrm{~s}$; y, finalmente, para la técnica de impedancia, se hizo un barrido en frecuencias desde $10 \mathrm{kHz}$ hasta $10 \mathrm{mHz}$. Este intervalo de frecuencias se dividió en 50 partes para obtener la respuesta de impedancia a frecuencias intermedias.

El conjunto a estudiar se encontraba contenido en una celda electroquímica de vidrio de tres electrodos, según muestra la figura 1 . Los ensayos se llevaron a cabo a temperatura ambiente, a aproximadamente $20^{\circ} \mathrm{C}$.

\section{RESULTADOS Y DISCUSIÓN}

En la figura 2 se muestra una curva de polarización con barrido directo e inverso de potencial del cobre en una solución al $3 \% \mathrm{NaCl}$, técnica sugerida por Pourbaix (16) para determinar la susceptibilidad de un material metálico a la corrosión por picaduras. Dicha curva de polarización presenta dos picos de corriente a 169 y $317 \mathrm{mV}$, que pueden atribuirse, el primero, a la formación de $\mathrm{Cu}_{2} \mathrm{O}$, y el segundo, a la formación de $\mathrm{Cu}_{2}(\mathrm{OH})_{3} \mathrm{Cl}$, según referenció Kato (17) para cobre en soluciones de $\mathrm{NaCl}$ a valores de $\mathrm{pH}$ ligeramente ácidos o con soluciones neutras. Posteriormente, se observa un incremento de corriente a $370 \mathrm{mV}$, correspondiente al potencial crítico de picado, aunque no de una manera muy significativa. En el regreso de potencial se presenta un fenómeno de histéresis con un incremento de corriente, que aunado a lo mencionado anteriormente indica que el cobre, en este medio, es susceptible a la corrosión por picaduras.

En las figuras 3 y 4 se aprecia claramente que existen picaduras en el cobre, pero no son profundas, lo que se refleja en un incremento real de la

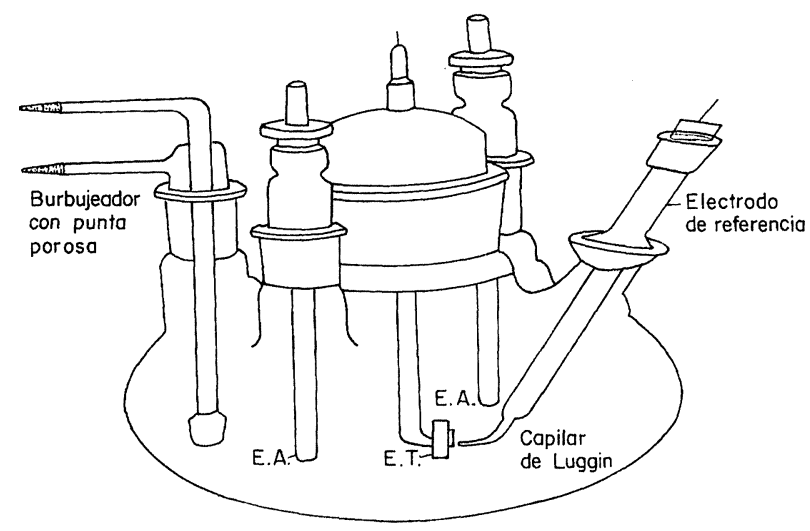

FIG. 1.- Celda para montaje con tres electrodos.

FIG. 1.-Three electrode electrochemical cell. 


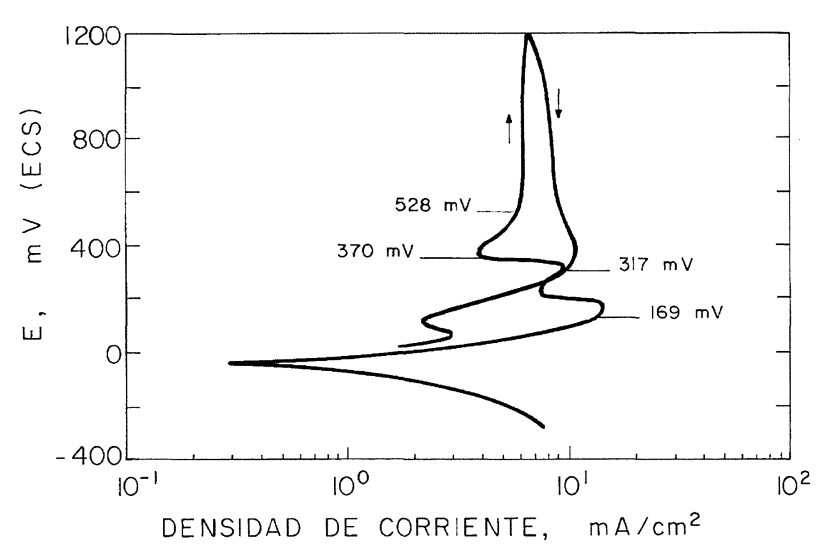

FIG. 2.- Curva de polarización de cobre en $\mathrm{NaCl}$ $3 \%, \mathrm{pH} 6,4$.

FIG. 2.-Polarization curve of copper in $3 \% \mathrm{NaCl}$ solution, $\mathrm{pH}$ 6,4.

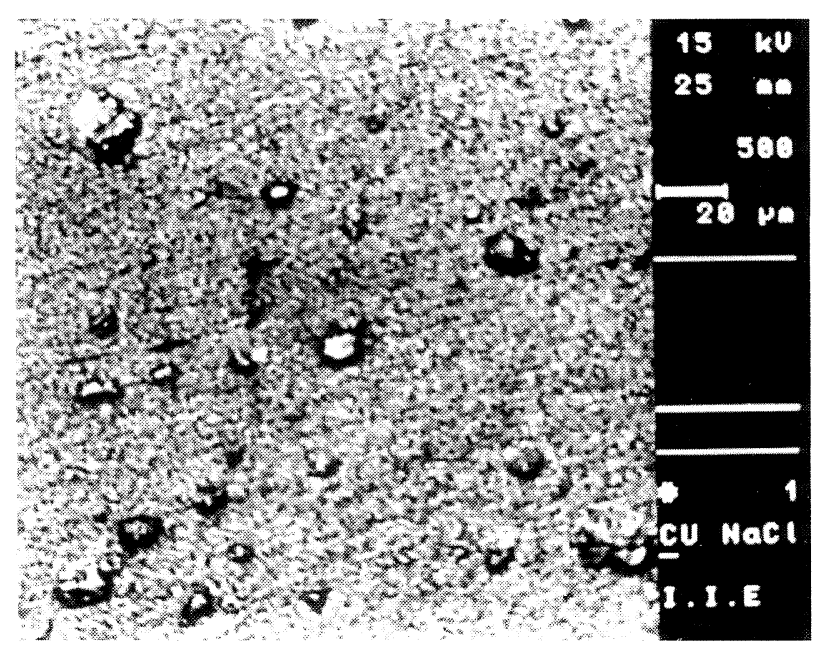

FIG. 3.- Micrografía de la superficie picada del cobre en $\mathrm{NaCl} 3 \%$. $\times 500$.

FIG. 3.- Micrograph of pitted surface of copper in $3 \% \mathrm{NaCl}$ solution. $\times 500$.

superficie y, por lo tanto, en un fenómeno de histéresis, con un potencial crítico de picado no muy evidente.

Esto se confirma con los ensayos de impedancia realizados (Figs. 5 y 6). Como se observa en la tabla I, que resume los resultados de los diagramas de impedancia obtenidos, en el segundo semicírculo capacitivo, de mayor tamaño, su constante de tiempo disminuye continuamente, indicando que se está formando a mayor velocidad un producto de corrosión de naturaleza insoluble. En cambio, se puede observar que en el semicírculo inductivo su constante de tiempo comienza con una disminución de su valor, pero al alcanzarse el potencial de $370 \mathrm{mV}$ este valor empieza de nuevo a incrementarse.

Esto quiere decir que, a potenciales inferiores a $370 \mathrm{mV}$, los productos de corrosión precipitan sobre

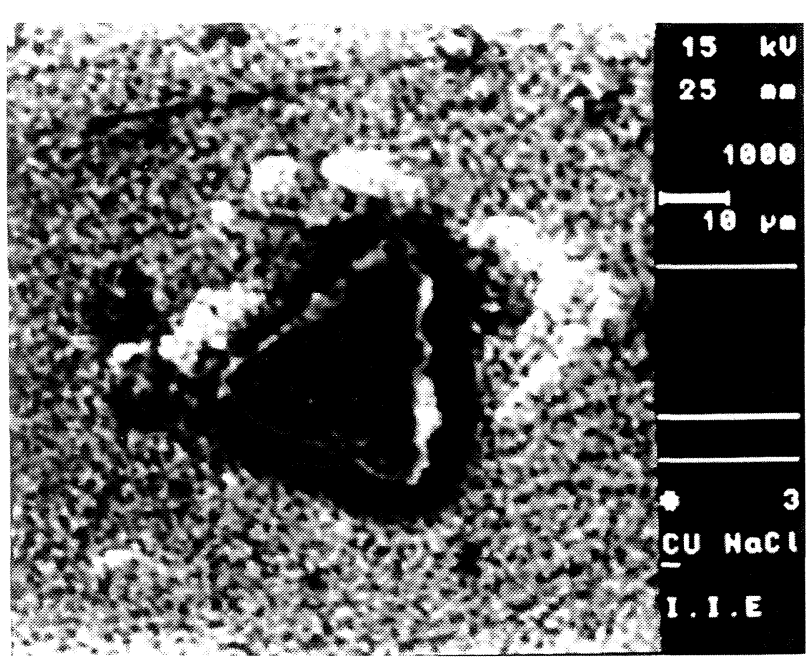

FIG. 4.- Micrografía de la picadura del cobre en $\mathrm{NaCl} 3 \% . \times 1.000$.

FIG. 4.-Micrograph of pitted surface of copper in $3 \% \mathrm{NaCl}$ solution. $\times 1,000$.

todá la superficie y, por tanto, en los diagramas de Nyquist presentan curvas cerradas (Fig. 5). En cambio, cuando se rebasa este potencial, la velocidad de formación de los productos insolubles se incrementa, mientras que la velocidad de precipitación de los mismos disminuye, motivando que, en consecuencia, el diagrama de Nyquist correspondiente presente una curva abierta (Fig. 6), sugiriendo que quedan descubiertas secciones del material que se corroen más intensamente. Pero esta diferencia de velocidades no es muy grande, debido a que no se aprecia dispersión en los puntos correspondientes a bajas frecuencias de los diagramas de Nyquist a potenciales superiores a los $370 \mathrm{mV}$.

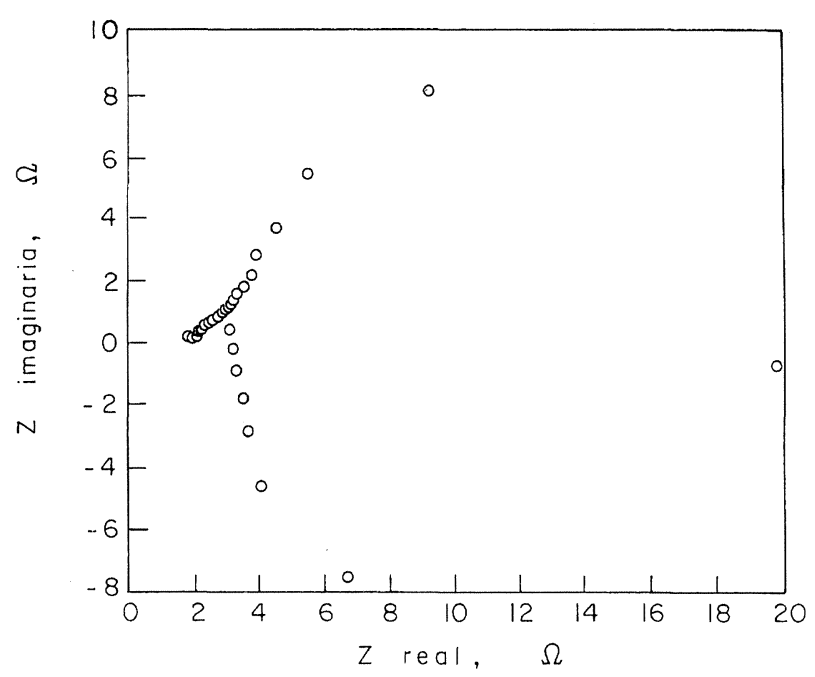

FIG. 5.- Diagrama de impedancia de cobre en $\mathrm{NaCl}, 3 \%$, a $250 \mathrm{mV}$.

FIG. 5.- Nyquist plot of copper in $3 \% \mathrm{NaCl}$, at $250 \mathrm{mV}$. 


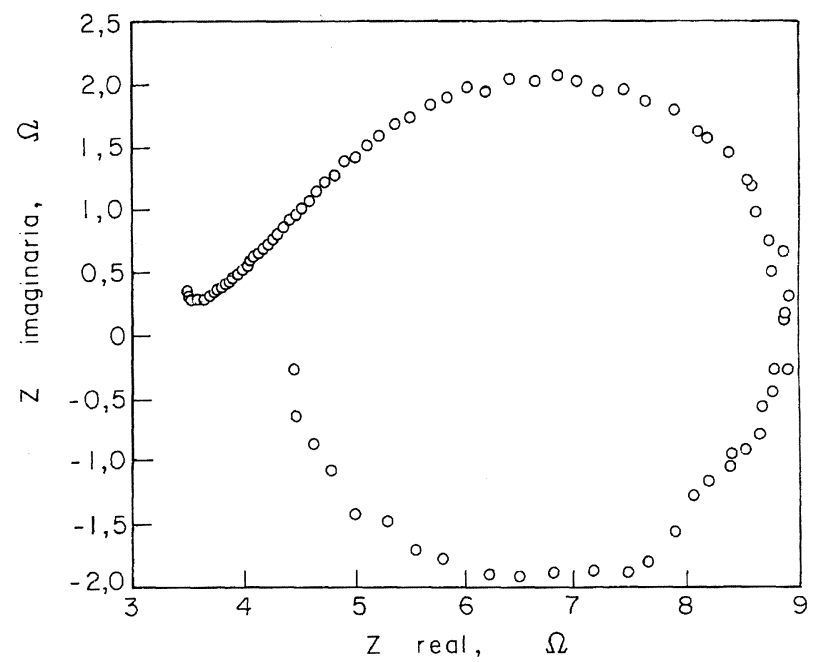

FIG. 6.- Diagrama de impedancia de cobre en $\mathrm{NaCl}, 3 \%$, a $550 \mathrm{mV}$.

FIG. 6.- Nyquist plot of copper in $3 \% \mathrm{NaCl}$, at $550 \mathrm{mV}$.

TABla I.- Constantes de tiempo de las pruebas de impedancia de cobre en $\mathrm{NaCl}(3 \%)$ a diferentes potenciales

TABLE I.- Time constants from impedance measurements of copper in $3 \% \mathrm{NaCl}$ solution at different potentials

\begin{tabular}{|c|c|c|c|}
\hline $\begin{array}{c}\text { Potencial, } \\
\mathrm{mV}\end{array}$ & $\begin{array}{c}\text { Constante de } \\
\text { tiempo 1 } \\
\text { semicírculo } \\
\text { capacitivo, } \\
\mathrm{s}\end{array}$ & $\begin{array}{c}\text { Constante de } \\
\text { tiempo 2 } \\
\text { semicírculo } \\
\text { capacitivo, } \\
\mathrm{s}\end{array}$ & $\begin{array}{c}\text { Constante de } \\
\text { tiempo del rizo } \\
\text { inductivo, } \\
\mathrm{s}\end{array}$ \\
\hline 250 & 0,04 & 1,44 & 2,51 \\
320 & 0,17 & 0,91 & 1,44 \\
350 & - & 0,75 & 1,20 \\
370 & - & 0,48 & 1,41 \\
400 & - & 0,17 & 2,02 \\
550 & - & 0,08 & 3,16 \\
\hline
\end{tabular}

Los ensayos de ruido en corriente del cobre en $\mathrm{NaCl}$ al $3 \%$ (Figs. 7, 8, 9, 10, 11 y 12) no manifies$\tan$ un incremento significativo de los niveles de ruido en alta frecuencia al ir incrementando el potencial, encontrándose esta intensidad alrededor de $-110 \mathrm{~dB}$.

Por otra parte, se aprecian cambios importantes en las componentes en baja frecuencia. Por ejemplo, se incrementa la intensidad de las componentes en baja frecuencia del potencial de $250 \mathrm{mV}$ (Fig. 7) de $-80 \mathrm{~dB}$ a $-40 \mathrm{~dB}$ al potencial de $320 \mathrm{mV}$ (Fig. 8). Esto se debe a que a $250 \mathrm{mV}$ se puede formar el primer producto de corrosión, el $\mathrm{Cu}_{2} \mathrm{O}$, como se aprecia en la figura 2. En cambio, a $320 \mathrm{mV}$ se obtiene la formación del segundo producto, el
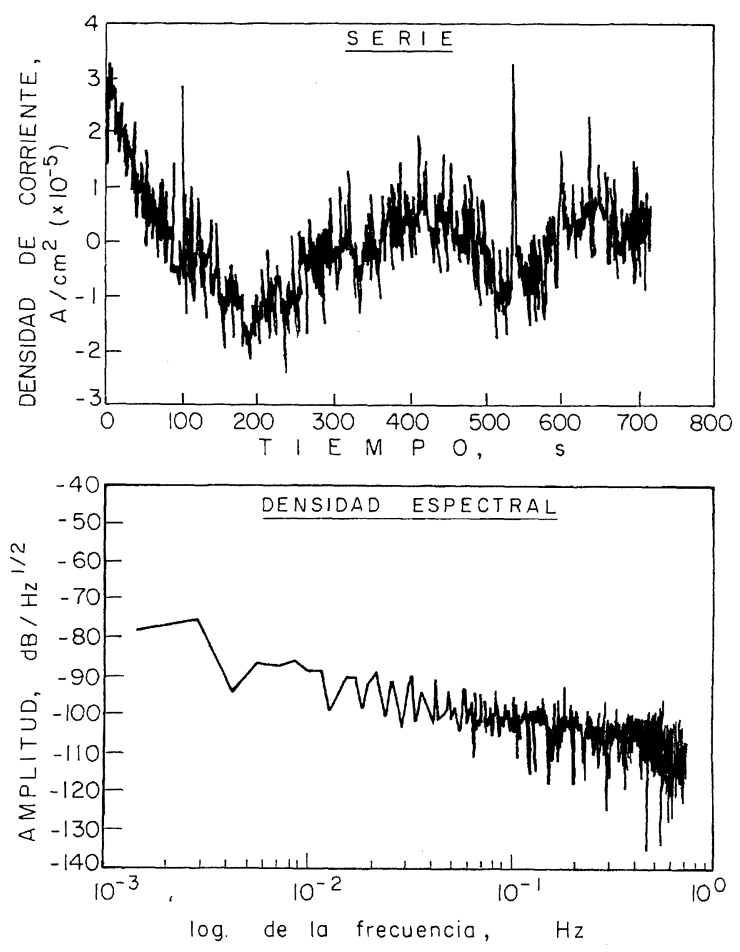

FIG. 7.- Ensayo de ruido en corriente de cobre en $\mathrm{NaCl}, 3 \%$, a $250 \mathrm{mV}$.

FIG. 7.-Electrochemical current noise test of copper in $3 \% \mathrm{NaCl}$, at $250 \mathrm{mV}$.
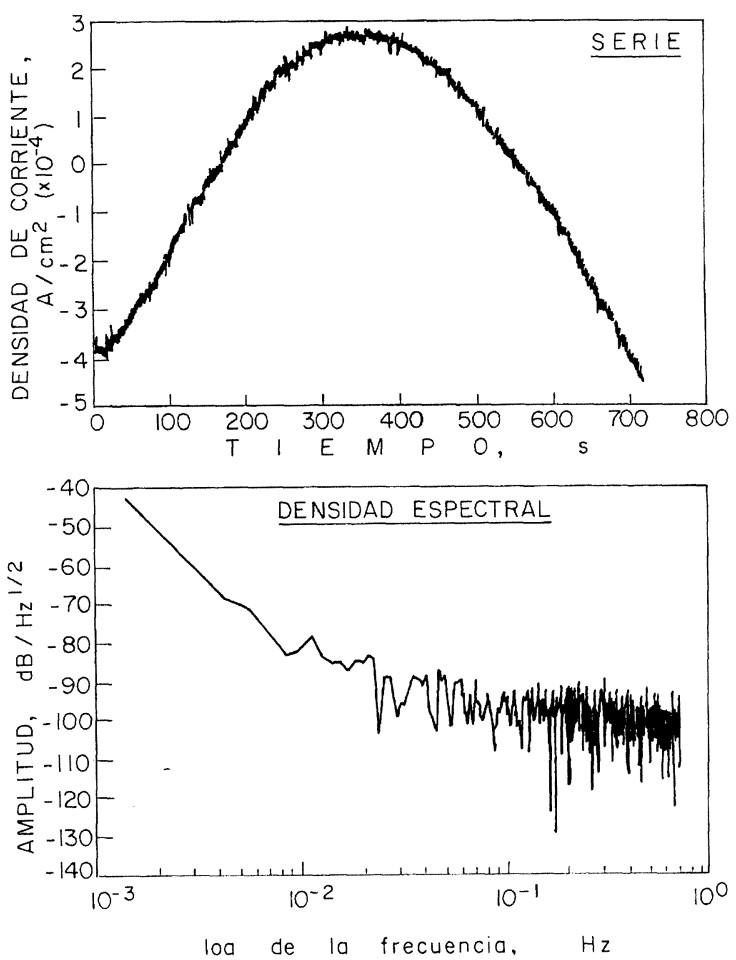

Fig. 8.- Ensayo de ruido en corriente de cobre en $\mathrm{NaCl}, 3 \%$, a $310 \mathrm{mV}$.

FIG. 8.-Electrochemical current noise test of copper in $3 \% \mathrm{NaCl}$, at $310 \mathrm{mV}$. 

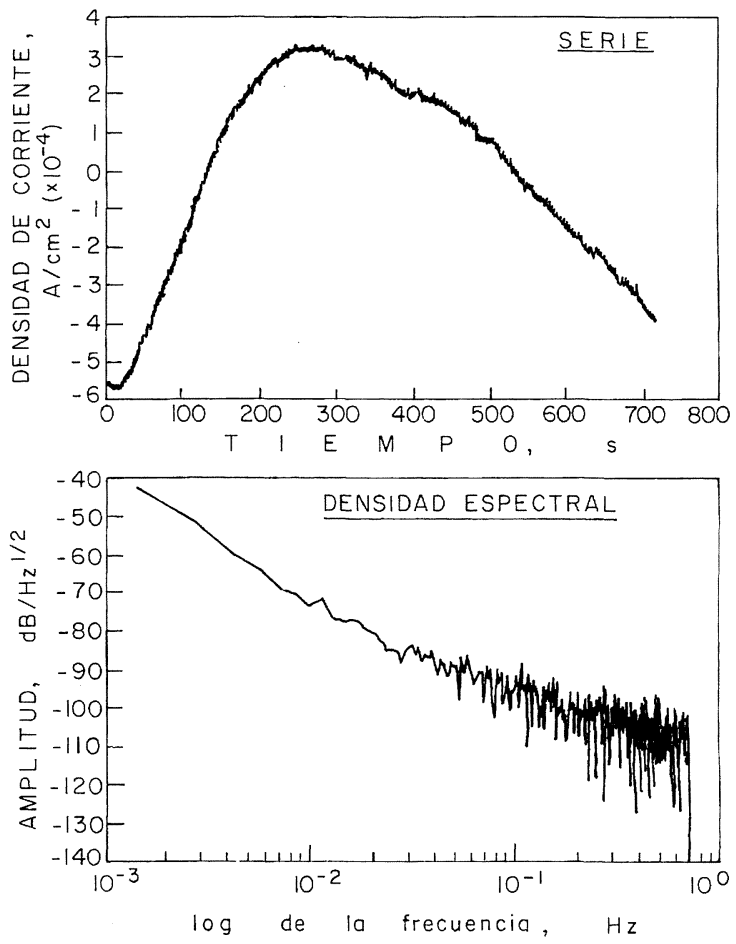

Fig. 9.- Ensayo de ruido en corriente de cobre en $\mathrm{NaCl}, 3 \%$, a $350 \mathrm{mV}$.

FIG. 9.-Electrochemical current noise test of copper in $3 \% \mathrm{NaCl}$, at $350 \mathrm{mV}$.
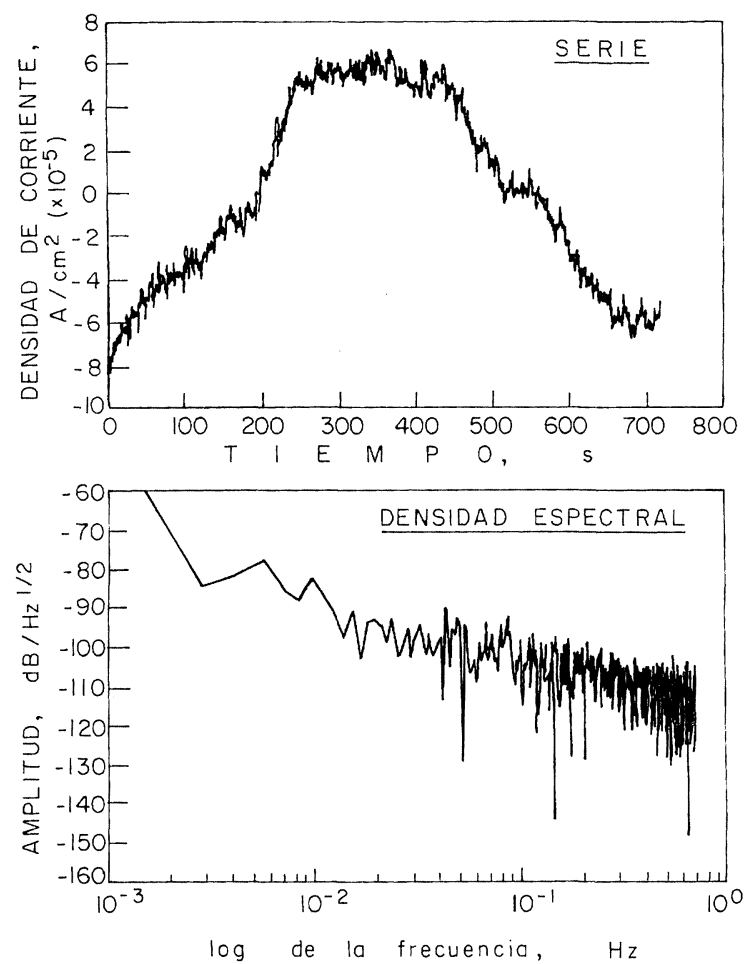

FIG. 10.- Ensayo de ruido en corriente de cobre en $\mathrm{NaCl}, 3 \%$, a $370 \mathrm{mV}$.

FIG. 10.-Electrochemical current noise test of copper in $3 \% \mathrm{NaCl}$, at $370 \mathrm{mV}$.
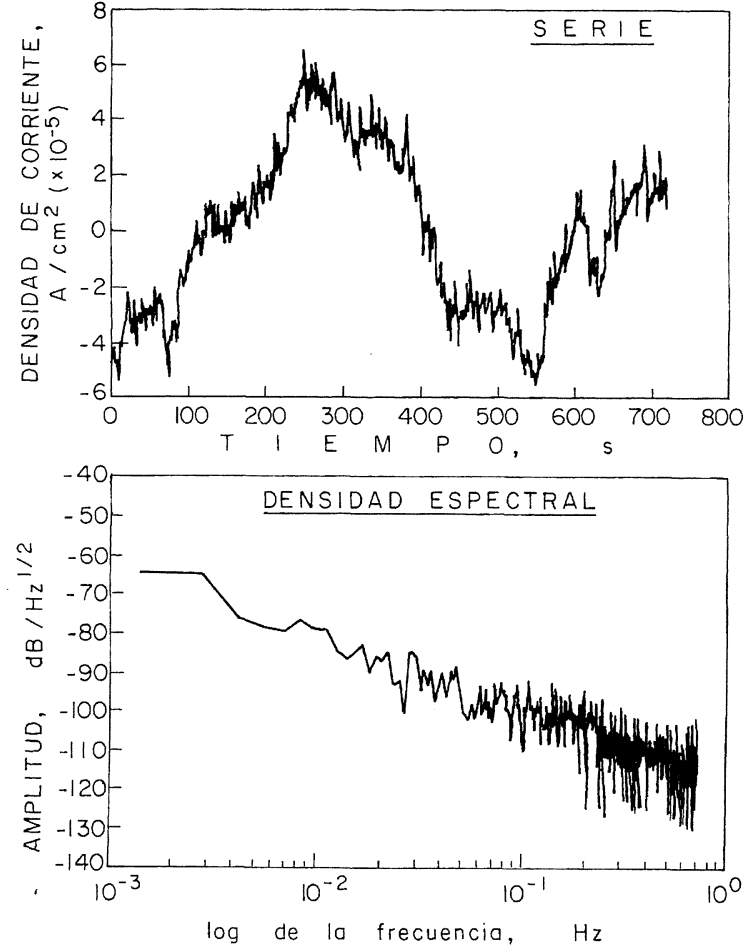

FIG. 11.- Ensayo de ruido en corriente de cobre en $\mathrm{NaCl}, 3 \%$, a $400 \mathrm{mV}$.

FIG. 11.-Electrochemical current noise test of copper in $3 \% \mathrm{NaCl}$, at $400 \mathrm{mV}$.
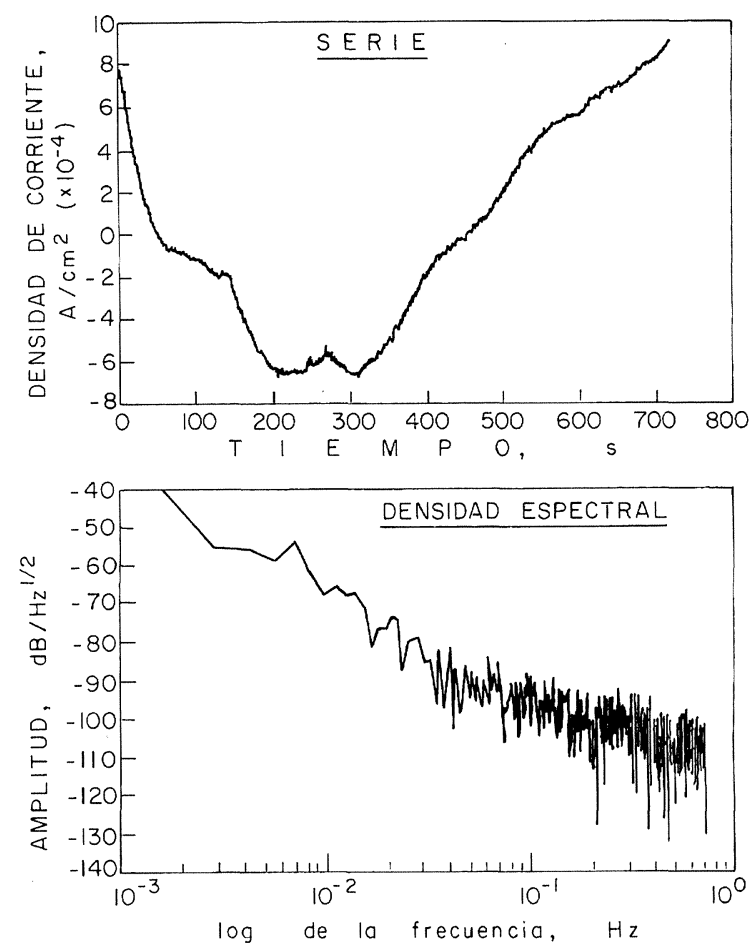

FIG. 12.- Ensayo de ruido en corriente de cobre en $\mathrm{NaCl}, 3 \%$, a $550 \mathrm{mV}$.

FIG. 12.-Electrochemical current noise test of copper in $3 \% \mathrm{NaCl}$, at $550 \mathrm{mV}$. 
$\mathrm{Cu}_{2}(\mathrm{OH})_{3} \mathrm{Cl}$, indicativa de la razón del cambio de nivel en la intensidad del ruido en baja frecuencia.

El nivel de ruido en baja frecuencia se mantiene a $-40 \mathrm{~dB}$ a potenciales de 320 y $350 \mathrm{mV}$ (Figs. 8 y 9) debido a que a estos potenciales es a los que se forma el segundo producto $\left(\mathrm{Cu}_{2}(\mathrm{OH})_{3} \mathrm{Cl}\right)$. En el momento de realizar la prueba de ruido en corriente del cobre en $\mathrm{NaCl}$ a $370 \mathrm{mV}$ (Fig. 10), el nivel de ruido a baja frecuencia disminuye a $-60 \mathrm{~dB}$, manteniéndose este mismo nivel al potencial de $400 \mathrm{mV}$ (Fig. 11) y, posteriormente, a un potencial de 500 $\mathrm{mV}$ (Fig. 12), se recupera nuevamente el nivel de $-40 \mathrm{~dB}$.

Estos cambios en el nivel de ruido a baja frecuencia indican que la formación de los productos está sufriendo modificaciones, lo que se confirma con los ensayos de impedancia a los potenciales de 370 y $400 \mathrm{mV}$, donde se observa una zona de transición y se inicia una disolución de los mismos en la superficie, posteriormente, aunque la disolución de los productos de corrosión se incrementa más (como se ve en la figura 6), el producto que continúa formándose es el $\mathrm{Cu}_{2}(\mathrm{OH})_{3} \mathrm{Cl}$, por la razón de que a dichos potenciales es el más estable $\mathrm{y}$, por lo tanto, el nivel de ruido a baja frecuencia regresa a su nivel original de $-40 \mathrm{~dB}$.

Estas observaciones llevan a proponer que el mecanismo de la corrosión por picaduras en el cobre en $\mathrm{NaCl}$ puede deberse a la formación de una capa protectora, y que en el momento en que esta capa protectora se disuelve en sitios localizados, favorece la formación de la picadura. De aquí que la velocidad de corrosión de la picadura con respecto al resto del material no sea significativamente mayor, produciendo, en consecuencia, una picadura de poca profundidad.

\section{CONCLUSIONES}

El mecanismo de la formación de picaduras en el cobre en solución de $\mathrm{NaCl}$ al $3 \%$ no parece estar asociado con la rotura y recuperación de la capa pasiva, como se puso de manifiesto en las señales de ruido en corriente, donde no se presentaron componentes en alta frecuencia como establecieron Uruchurtu y col. (3) para mecanismos de esta naturaleza.

Se puede presumir que, a partir de la información proporcionada por la técnica de impedancia, el origen de la iniciación de la picadura en este sistema pudiera deberse a una disolución localizada de la capa pasiva, lugar en el que tiene origen la picadura.

\section{REFERENCIAS}

(1) MAY, R. J. Inst. Met., 32, 1953: 65-68.

(2) Gabrielli, C., Huet, F., Keddam, M., Oltra, R. y Pallota, C. Passivity of Metals and Semiconductors. Ed. Elsevier. Amsterdam, 1983: 293.

(3) Uruchurtu, J. y Dawson, J.L. Corrosion (NACE), 43 (1), 1987: 19-25.

(4) Kolotyrkin, Ya.M. Corrosion (NACE), 19 (8), 1963: 261-268.

(5) Uhlig, H.H. Corros. Sci., 19, 1979: 777-791.

(6) Kolotyrkin, Ya.M. J. Electrochem. Soc., 108 (3), 1961: 209-217.

(7) HOAR, T.P. Corros. Sci., 7, 1967: 314-318.

(8) Heine, M.A., KeIR, D.S. y Pryor, M.J. J. Electrochem. Soc., 112 (1), 1965: 24-32.

(9) Hoar, T.P., Mears, D.C. y Rothwell, G.P. Corros. Sci., 5, 1965: 279-289.

(10) Richardson, J.A. y Wood, G.C. Corros. Sci., 10, 1970: 313.

(11) Sato, N. Electrochim. Acta, 19, 1971: 1.683-1.692.

(12) Vetter, H.J. y Strenhblow, H.H. Proc. Conf. on Localized Corrosion. Eds. R.W. Staehle, B.F. Brown, J. Kruger y A. Agrawal. Ed. NACE. Houston, 1974: 240.

(13) Galvele, J.R. J. Electrochem. Soc., 123 (4), 1976: 464474.

(14) Szklarska-Smialowska, Z. Br. Corros. J., 10, 1975: 11 15.

(15) SzKLarska-SmialowsKa, Z. Pitting Corrosion of Metals. Ed. NACE. Houston, 1986: 41.

(16) Pourbaix, M. Proc. Conf. on Localized Corrosion. Eds. R.W. Staehle, B.F. Brown, J. Kruger y A. Agrawal. Ed. NACE. Houston, 1974: 12.

(17) Kato, C. y Pickering, H.W. J. Electrochem. Soc., 131 (6), 1984: 1.219-1.224. 